\title{
Estudio de la incorporación de iones de Er y Nd en galio antimonio crecido por el método Bridgman
}

\author{
J.L.PLAZA ${ }^{1}$, P. HIDALGO ${ }^{2}$, J. PIQUERAS ${ }^{2}$, E. DIÉGUEZ ${ }^{1}$ \\ ${ }^{1}$ Departamento de Física de Materiales, Universidad Autónoma de Madrid, 28049, Cantoblanco. \\ ${ }^{2}$ Departamento de Física de Materiales, Universidad Complutense de Madrid, 28040, Madrid.
}

\begin{abstract}
En este trabajo se ha realizado el estudio de cristales de GaSb crecidos por el método Bridgman y dopados con Er y Nd con distintas concentraciones. Se han realizado análisis de absorción atómica pudiéndose obtener el coeficiente de segregación efectivo de ambos dopantes a lo largo de la dirección de crecimiento del material. Mediante medidas de efecto Hall se ha determinado el tipo de portadores mayoritarios (huecos) así como los valores de la movilidad, la densidad de portadores y la resistividad para cada una de las concentraciones. Los análisis de dispersión de energías de rayos X (EDAX) y de microscopio electrónico de barrido (SEM) han demostrado la presencia de agregados formados por los iones de tierras raras y $\mathrm{Sb}$ para las concentraciones de dopante más altas. La reducción de los defectos nativos tales como las vacantes de Ga y Ga en posición de $\mathrm{Sb}$ por los iones de Er ha sido también demostrado a través de análisis de catodoluminiscencia.
\end{abstract}

\section{Palabras clave: Antimoniuro de galio, tierras raras.}

\section{Study of the incorporation of the Er and Nd Ions in Gallium Antimonide grown by the Bridgman method}

In this work the study of GaSb crystals grown by the Bridgman method doped with Er and Nd with different concentrations has been carried out. Atomic absorption analysis have been developed for obtaining the effective segregation coefficient of the two dopants along the growth direction of the material. The resistivity, mobility and density of carriers were obtained by the van der Pauw technique for the different dopant concentrations. The energy dispersive $\mathrm{X}$ ray analysis (EDX) and the scanning electron microscope (SEM) have demonstrated the presence of precipitates made from $\mathrm{Sb}$ and rare earth elements for the highest dopant concentrations. Cathodoluminescence (CL) analysis have proved that the rare earth elements have a reduction effect of native defects in GaSb.

Keywords : Gallium antimonide, rare earths.

\section{INTRODUCCION}

Los semiconductores dopados con tierras raras (TR) se han presentado recientemente como materiales de gran interés científico debido a sus prometedoras aplicaciones en el campo de la optoelectrónica y las telecomunicaciones. Los dispositivos basados en este tipo de materiales presentan una gran estabilidad de la luminiscencia frente a cambios en la temperatura que además no se ve afectada prácticamente por el tipo de matriz semiconductora utilizada.

Fundamentalmente el principal interés de los semiconductores dopados con TR se debe a la posibilidad de fabricación de dispositivos electroluminiscentes que son capaces de combinar tanto las propiedades luminiscentes de los iones de tierras raras como las propiedades electrónicas de los semiconductores (1). Cuando se introducen elementos de TR como iones trivalentes en matrices sólidas, estos presentan transiciones ópticas de tipo atómico. Estas transiciones se deben a la capa incompleta $4 \mathrm{f}$ que se encuentra apantallada por los orbitales llenos mas externos $5 s^{2}$ y $5 p^{6}$. Cuando el ion se encuentra en estado libre, estas transiciones intra- $4 \mathrm{f}$ son prohibidas debido a la conservación de la paridad. Por otra parte, cuando los cuando los iones de TR se encuentran en el interior de un sólido dichas transiciones son permitidas debido a la mezcla de estados de paridad opuesta producida por los campos eléctricos locales presentes en el interior de la matriz (2). De esta forma los iones de TR interactúan débilmente con la matriz en la que se encuentran y por tanto la longitud de onda de la emisión intra-4f es prácticamente independiente del material del que está compuesta la matriz así como de la temperatura del entorno.

En la literatura se han presentado diversos métodos utilizados para obtener la incorporación de iones de TR en semiconductotres III-V durante el proceso de crecimiento en volumen (3-5), abriendo nuevas posibilidades para la fabricación de dispositivos optoelectrónicos. En particular el ion $\mathrm{Er}^{3+}$ es uno de los mas interesantes debido al hecho de que su transición del estado ${ }^{4} \mathrm{I}_{13 / 2}$ al estado ${ }^{4} \mathrm{I}_{15 / 2}$ se produce a una longitud de onda de $1540 \mathrm{~nm}$. que coincide con aquella para la cual las pérdidas durante la transmisión de señales a través de las fibras ópticas basadas en silicatos son mínimas.

Además del ion Er, se han demostrado otras propiedades interesantes de distintos iones de TR cuando se incorporan en el interior de semiconductores III-V (6-7). En particular el ion $\mathrm{Nd}^{3+}$ resulta ser una buena elección a la hora de controlar la carga superficial en las interfases semiconductor-dieléctrico (8).

En este trabajo se han estudiado las características de segregación de Er y $\mathrm{Nd}$ en antimoniuro de galio ( $\mathrm{GaSb}$ ) crecido mediante el método Bridgman así como los cambios en las propiedades eléctricas y de la estructura superficial de diversas obleas sometidas a tratamientos de pulido mecánico y ataque químico. 


\section{PROCEDIMIENTO EXPERIMENTAL}

Para la realización de este trabajo se crecieron siete lingotes de GaSb puro y dopado con diversas concentraciones de Er y $\mathrm{Nd}$ mediante el método Bridgman, todos ellos con unas dimensiones de $60 \mathrm{~mm}$. de largo por $12 \mathrm{~mm}$. de diámetro. Para ello se han utilizado como materiales de partida elementos en estado metálico de alta pureza ( $\mathrm{Ga}, \mathrm{Sb} 6 \mathrm{~N}$ y Er, Nd 3N). Tanto las concentraciones iniciales de dopante de los lingotes obtenidos como su nomenclatura se encuentran descritas en la Tabla I. A partir de este momento nos referiremos a las muestras mediante el número del lingote del que provengan y en caso necesario especificaremos la región de la cual se extrajeron. Los crisoles en el interior de los cuales se ha realizado el crecimiento constan básicamente de ampollas de cuarzo de alta calidad y selladas al vacío a $10^{-6}$ Torr las cuales fueron previamente grafitadas mediante un proceso de pirólisis de acetona a $1000{ }^{\circ} \mathrm{C}$ con el fin de evitar la adherencia del material a las paredes interiores.

El crecimiento se realizó a una velocidad de $3 \mathrm{~mm} / \mathrm{h}$ en el interior de un horno Bridgman oscilante de una sola zona calefactora cuyo gradiente térmico es de $35{ }^{\circ} \mathrm{C} / \mathrm{cm}$. Pueden encontrarse mas detalles sobre esta técnica en la referencia (9). Para realizar los diversos estudios se cortaron diversas obleas según planos perpendiculares a la dirección de crecimiento.

Para obtener la concentración de dopante tanto en la dirección radial como axial se ha utilizado la técnica de espectrometría de absorción atómica (AAS). El material analizado se extrajo tanto de la región central como externa de diversas obleas cortadas a lo largo del lingote. El aparato utilizado para tal efecto fue un Perkin Elmer 3110. El pulido mecánico se realizó mediante el uso de polvo de alúmina de 5, 1, 0.3 y 0.05 $\mathrm{mm}$. Después de este proceso algunas de las muestras fueron sometidas a ataque químico durante $30 \mathrm{seg}$. con una solución tipo $\mathrm{CP} 4\left(1 \mathrm{CH}_{3} \mathrm{COOH}\right.$ : $5 \mathrm{HF}: 9 \mathrm{HNO}_{3}$ : $\left.10 \mathrm{H}_{2} \mathrm{O}\right)$ seguido de la inmersión de las muestras en metanol. Para la medida de la resistividad, movilidad y densidad de portadores se ha utiliza-
TABLA I. CONCENTRACIÓN INICIAL DE DOPANTE $\left(x 10^{19} \mathrm{~cm}^{-3}\right)$ DE LOS DISTINTOS LINGOTES.

\begin{tabular}{|c|c|c|c|c|c|c|}
\hline $\begin{array}{r}\text { Lingote 24 } \\
\text { GaSb puro }\end{array}$ & $\begin{array}{c}\text { Lingote 25 } \\
\text { GaSb }: \mathrm{Er}\end{array}$ & $\begin{array}{r}\text { Lingote 26 } \\
\mathrm{GaSb}: \mathrm{Er}\end{array}$ & $\begin{array}{r}\text { Lingote 27 } \\
\mathrm{GaSb}: \mathrm{Er}\end{array}$ & $\begin{array}{c}\text { Lingote 28 } \\
\mathrm{GaSb}: \mathbf{N d}\end{array}$ & $\begin{array}{c}\text { Lingote 29 } \\
\mathrm{GaSb}: \mathbf{N d}\end{array}$ & $\begin{array}{c}\text { Lingote 30 } \\
\mathrm{GaSb}: \mathbf{N d}\end{array}$ \\
\hline & 1.0 & 2.0 & 8 & 0.6 & 2.4 & 9.6 \\
\hline
\end{tabular}

do la técnica de Van der Paw a temperatura ambiente utilizándose campos magnéticos de hasta 7 KGauss. Los contactos óhmicos requeridos se realizaron mediante pequeños puntos de In. El correcto comportamiento óhmico de estos contactos se verificó previamente a la realización de las medidas mediante la obtención de las curvas I-V.

Se han realizado también medidas de microscopía electrónica de barrido (MEB) así como análisis químico en la superficie de las muestras mediante análisis de dispersión de energía de rayos $X$ (EDAX). Para ello se ha utilizado un equipo Philips modelo XL30 que lleva acoplado un analizador EDAX modelo DX4i. Los voltajes de aceleración utilizados fueron de $25 \mathrm{kV}$. También se han realizado estudios de catodoluminiscencia mediante un equipo Hitachi S-2500 SEM a $77 \mathrm{~K}$ también con voltajes aceleradores de $25 \mathrm{kV}$.

\section{RESULTADOS Y DISCUSION}

\subsection{Estudio de la segregación axial}

Con el fin de obtener los perfiles de concentración de dopante y obtener cierta información sobre la incorporación de $\mathrm{Nd}$ en la matriz de $\mathrm{GaSb}$, se han realizado análisis de absorción atómica de los lingotes con más altas concentraciones de dopante (lingotes 26, 27, 29 y 30), ya que las concentraciones mas bajas se encontraban por debajo del límite de detección del aparato. Estos perfiles de concentración frente a la fracción
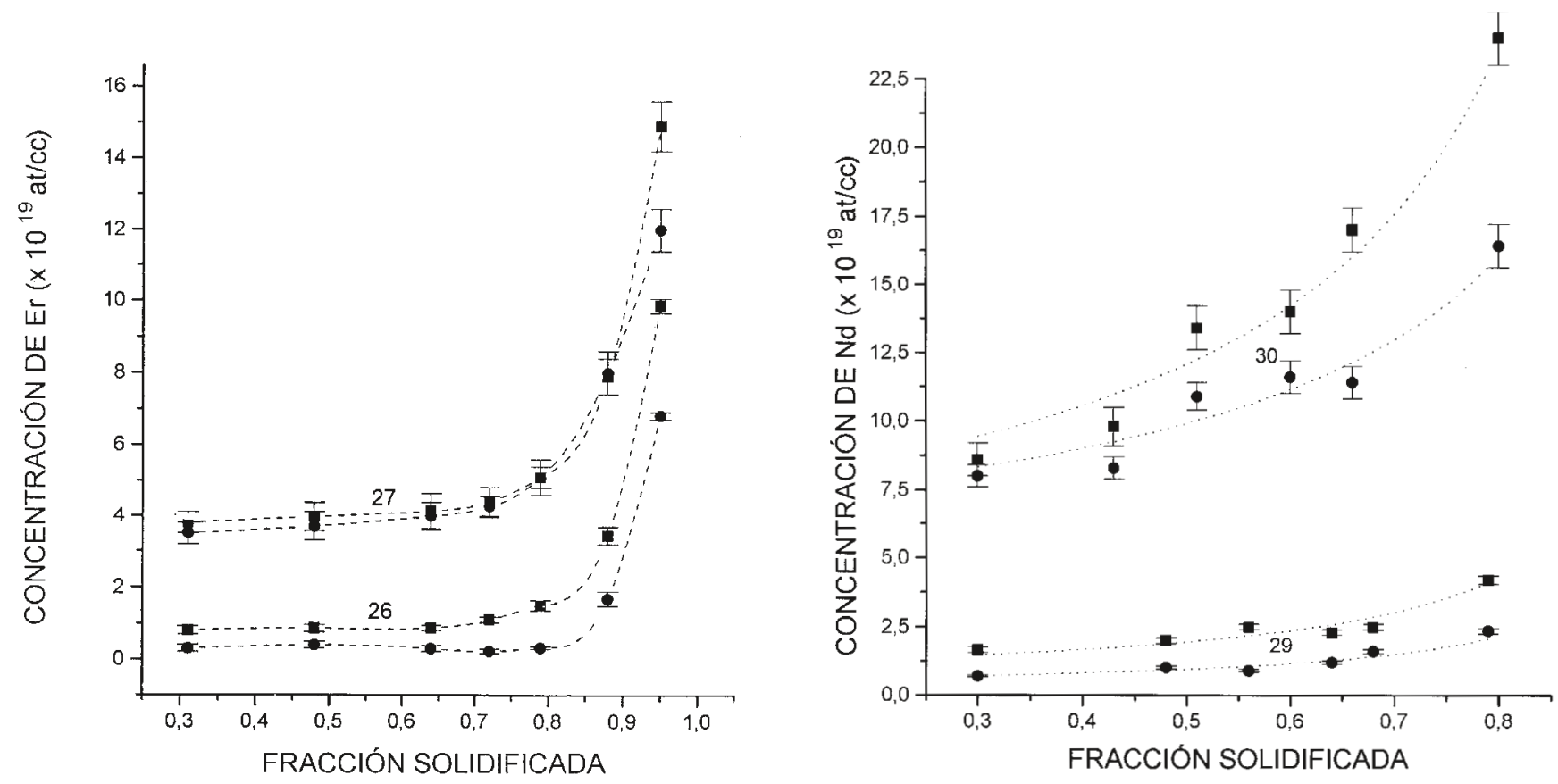

Figura 1. Perfiles de concentración frente a la fracción solidificada para (a) lingotes dopados con Er y (b) lingotes dopados con Nd. los símbolos $(\bullet)$ representan medidas tomadas en el centro de las muestras mientras que los símbolos $(\boldsymbol{\square})$ se refieren al análisis en la periferia. 
TABLA II. VALORES DEL COEFICIENTE DE SEGREGACIÓN OBTENIDOS A PARTIR DEL AJUSTE MEDIANTE LA LEY DE SCHEIL DE LOS DATOS EXPERIMENTALES PRESENTADOS EN LA FIGURA 1.

\begin{tabular}{|c|c|c|}
\hline LINGOTE & $\mathbf{K}_{\text {eff }}$ (Centro) & $\mathbf{K}_{\text {eff }}$ (Periferia) \\
\hline 26 & $0.09 \pm 0.03$ & $0.08 \pm 0.02$ \\
27 & $0.36 \pm 0.03$ & $0.44 \pm 0.02$ \\
29 & $0.10 \pm 0.02$ & $0.14 \pm 0.02$ \\
30 & $0.27 \pm 0.04$ & $0.48 \pm 0.05$ \\
\hline
\end{tabular}

de masa solidificada se presentan en la figura 1a y $1 b$, en los cuales no se ha tenido en cuenta la concentración anómalamente alta del extremo y debida al estado transitorio final. Como puede observarse, en ambos casos la concentración de dopante es aproximadamente constante durante las primeras tres cuartas partes solidificadas del lingote. Este comportamiento es el mismo tanto en el extremo como en la periferia.

Por otra parte, para el caso de la concentración más alta de $\mathrm{Nd}$ se observa que la concentración es creciente a lo largo de todo el lingote. En el caso del lingote 26 la diferencia entre las concentraciones en el centro y la periferia es más alta que en el caso del lingote 27. Por otra parte, para el caso de los lingotes dopados con Nd puede observarse que las diferencias de concentración entre el centro y la periferia son prácticamente del mismo orden y en el caso del lingote más altamente dopado (lingote 30), estas diferencias van aumentando a medida que nos acercamos a la última zona solidificada. En todos los casos se ha observado que la concentración en el centro de las obleas es siempre ligeramente menor que la concentración en los extremos, debido probablemente al arrastre de material desde la zona central hacia los laterales a consecuencia de corrientes de convección.

Los puntos experimentales presentados en la figura 1 pueden ajustarse a la ley de Scheil que relaciona la concentración de dopante en el sólido con la concentración inicial en el fundido y el coeficiente de segregación efectivo de la siguente forma $(11,12)$ :

$$
C_{s}=K_{e f f} C_{0}(1-g)^{K_{e f f}-1}
$$

donde $C_{S}$ es la concentración de dopante en el sólido, $K_{\text {eff }}$ es el coeficiente de segregación efectivo, $C_{0}$ es la concentración inicial en el fundido y $g$ es la fracción solidificada. A partir de la expresión (1) podemos obtener la siguiente expresión linealizada :

$$
\ln C_{s}=\left(K_{e f f}-1\right) \ln (1-g)+\ln \left(K_{e f f} C_{0}\right)
$$

de

donde puede obtenerse fácilmente $K_{\text {eff }}$ Los resultados obtenidos de este ajuste se muestran en la tabla II. Por comparación de estos resultados tenemos que tanto en el caso del Er como del Nd los coeficientes de segregación aumentan con la concentración para un mismo dopante siendo mayores en la periferia que en el centro para concentraciones altas mientras que se mantienen prácticamente constantes a bajas concentraciones. Este comportamiento podría explicarse si recurrimos a la dependencia de $K_{\text {eff }}$ con el espesor de la capa límite que a su vez depende de la concentración de dopante. De esta forma, utilizando la teoría BPS y asumiendo mezcla parcial de dopante en el fundido, $K_{e f f}$ vendrá dado por la siguiente expresión (10) :

$$
K_{\text {eff }}=\frac{k}{k+(1-k) \exp \left(-\frac{\delta_{s f} V_{I}}{D}\right)}
$$

siendo $k$ el coeficiente de segregación en el equilibrio, $V_{I}$ es la velocidad de crecimiento, $D$ es el coeficiente de difusión y $\delta_{s f}$ es el grosor de la capa límite de soluto. En la expresión anterior puede observarse que al aumentar $\delta_{s f}$ crece el valor de $\quad K_{e f f}$. A la vista de los resultados presentados en la tabla I, esto significa que en el caso de los lingotes 27 y 30, el grosor de la capa límite de soluto es mayor en la periferia que en el centro debido a un exceso de dopante en esta última región inducido por el flujo convectivo tal y como hemos mencionado previamente.

\subsection{Estudio de la superficie mediante MEB-EDAX}

Se ha realizado un estudio de la superficie mediante SEM sobre diversas muestras tomadas de la región central del lingote. En las muestras analizadas que han sido previamente pulidas no se ha observado ninguna indicación de daño mecánico. Únicamente la muestra 27 con la mayor concentración de Er ha mostrado claramente precipitados triangulares de aproximadamente $10 \mu \mathrm{m}$ de tamaño medio incluso después
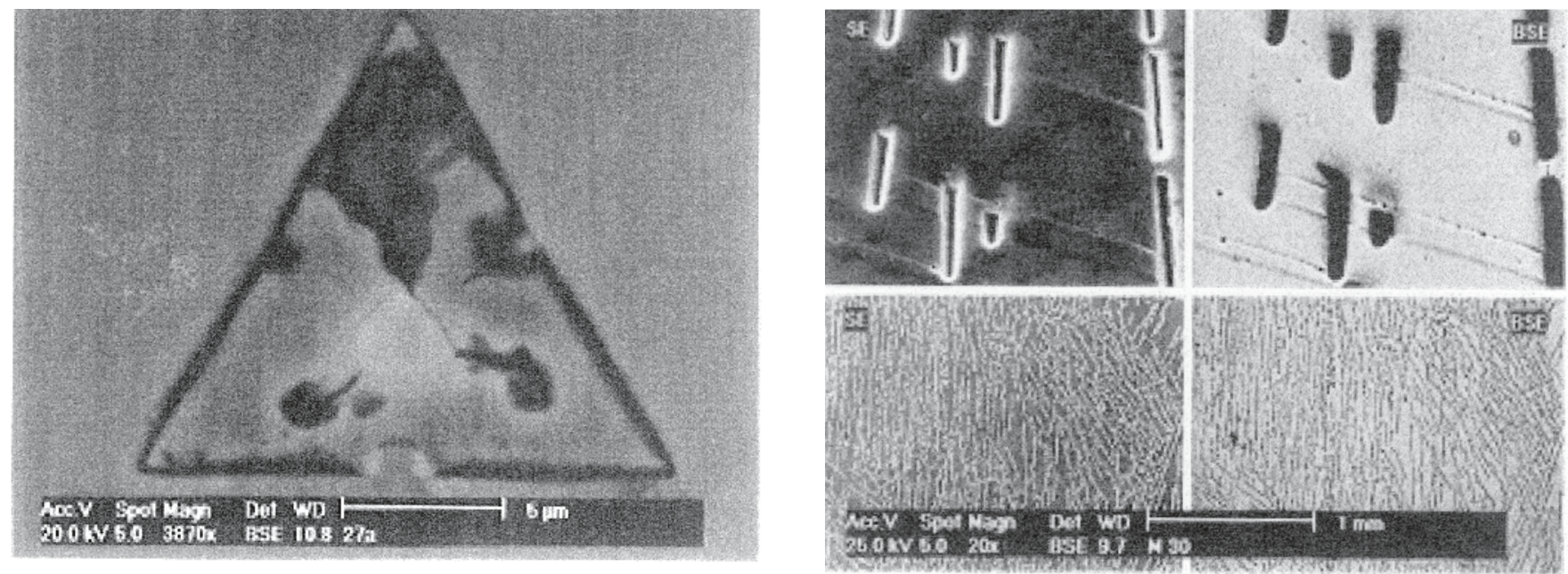

Figura 2. (a) Imagen mediante MEB en modo de electrones retrodispersados de un precipitado observado en muestras pulidas de Er con alta concentración (lingote 28). (b) Imagen mediante MEB de diversos precipitados observados en el lingote 30. 
del proceso de pulido. Uno de estos precipitados se muestra en detalle en la figura 2a. La formación de estos precipitados que, como veremos mas adelante, presentan una gran concentración de Sb y Er, podría explicarse teniendo en cuenta la tendencia de los elementos de tierras raras a formar compuestos junto con los elementos del grupo $\mathrm{V}$ de la tabla periódica y que reciben el nombre de pnictidos (13). La existencia de precipitados no ha sido detectada en el caso de las muestras pulidas y dopadas con $\mathrm{Nd}$.

La composición química ha sido analizada tanto en el interior como en el exterior de estos precipitados por EDAX dando lugar a la siguiente composición : $\mathrm{Sb}_{\text {int }}=35.06 \%$ at., $\mathrm{Ga}_{\text {int }}=$ $53.31 \%$ at., $\mathrm{Er}_{\text {int }}=11.63 \%$ at., $\mathrm{Sb}_{\text {ext }}=40.45 \%$ at., $\mathrm{Ga}_{\text {ext }}=59.06$ $\%$ at., $\mathrm{Er}_{\text {ext }}=0.49 \%$ at. donde en este caso los subíndices "int" y "ext" se refieren a zonas analizadas en el interior y en el exterior de los precipitados respectivamente. Como puede observarse, la concentración de Er en el interior es mucho más alta que en el exterior. Por otra parte, las proporciones de Ga y $\mathrm{Sb}$ se encuentran lejos de la estequiometría notándose una mayor abundancia de $\mathrm{Ga}$ respecto al $\mathrm{Sb}$ que se ha detectado también en las muestras puras. Esto podría explicar la concentración anómalamente alta de huecos en el GaSb puro bien conocida en la literatura y que se encuentra entorno a $10^{17} \mathrm{~cm}^{-3}$ y que se asocia fundamentalmente a antisitios de Ga que ocupan posiciones de $\mathrm{Sb}$ (14).

El ataque químico ha revelado el mismo tipo de precipitados en el caso de las muestras dopadas con Er. El aspecto de estos precipitados es básicamente el mismo pero con fronteras mucho más ensanchadas debido a un efecto mucho más acentuado del ataque en esta región debido a que el ácido queda confinado en el interior de las grietas que separan los precipitados del material circundante. Por otra parte se han observado también precipitados de formas alargadas en las muestras dopadas con alta concentración de Nd (lingote 30) y atacadas químicamente. Diversas imágenes de estos precipitados y a diferentes escalas se presentan en la figura $2 \mathrm{~b}$. El análisis EDAX realizado en el interior y el exterior de precipitados ha resultado en la siguiente composición : $\mathrm{Sb}_{\text {int }}=44.47 \%$ at., $\mathrm{Ga}_{\text {int }}$ $=52.04 \%$ at., $\mathrm{Nd}_{\text {int }}=3.49 \%$ at., $\mathrm{Sb}_{\text {ext }}=47.93 \%$ at., $\mathrm{Ga}_{\text {ext }}=51.20$ $\%$ at, $\mathrm{Nd}_{\text {ext }}=0.87 \%$ at. Debe indicarse que aunque en este caso la concentración de $\mathrm{Nd}$ en el interior de los precipitados es
Tabla III. Medidas de la resistividad, movilidad y densidad de portadores obtenidas de las muestras provenientes de la región central de los lingotes.

\begin{tabular}{|c|c|c|c|}
\hline LINGOTE & $\begin{array}{l}\text { Resistividad } \\
\quad(\Omega \cdot \mathrm{cm})\end{array}$ & $\begin{array}{c}\text { Movility } \\
\left(\mathrm{cm}^{2} / \mathrm{V} \cdot \mathrm{seg}\right)\end{array}$ & $\begin{array}{l}\text { Densidad de huecos } \\
\qquad\left(\times 10^{17} \mathrm{~cm}^{-3}\right)\end{array}$ \\
\hline Puro & 0.0900 & 550 & 1.0 \\
\hline 25 & 0.035 & 330 & 5.0 \\
\hline 26 & 0.018 & 280 & 12 \\
\hline 27 & 0.0017 & 180 & 250 \\
\hline 28 & 0.0854 & 410 & 1.8 \\
\hline 29 & 0.0714 & 370 & 2.4 \\
\hline 30 & 0.0273 & 230 & 10.0 \\
\hline
\end{tabular}

mucho menor que en el caso del Er, la composición de Ga y Sb está mucho mas cerca de la estequiometría.

\subsection{Medidas eléctricas mediante la técnica de Van der Paw}

Los valores de la resistividad, movilidad y densidad de portadores para las muestras anteriormente analizadas y extraídas de la región central de los lingotes se presentan en la tabla III. Como puede observarse, existe una clara disminución de la resistividad a medida que aumenta la concentración de dopante. Este comportamiento podría explicarse si suponemos que los iones de tierras raras ocupan posiciones intersticiales. Por otra parte la posición de los iones de tierras raras en matrices semiconductoras III-V no está muy clara y por tanto debe realizarse un estudio mas profundo sobre este tema.

\subsection{Análisis de Catodoluminiscencia}

El espectro de CL para muestras de GaSb dopadas con Er ha sido ya previamente publicado en la literatura (7), encontrándose un incremento de la intensidad total de CL produciéndose además cambios espectrales respecto a muestras de GaSb no dopadas. Se ha observado además una gran reducción de aceptores con el aumento en la concentración de Er. Dicha reducción llega a ser máxima para concentraciones de dopante
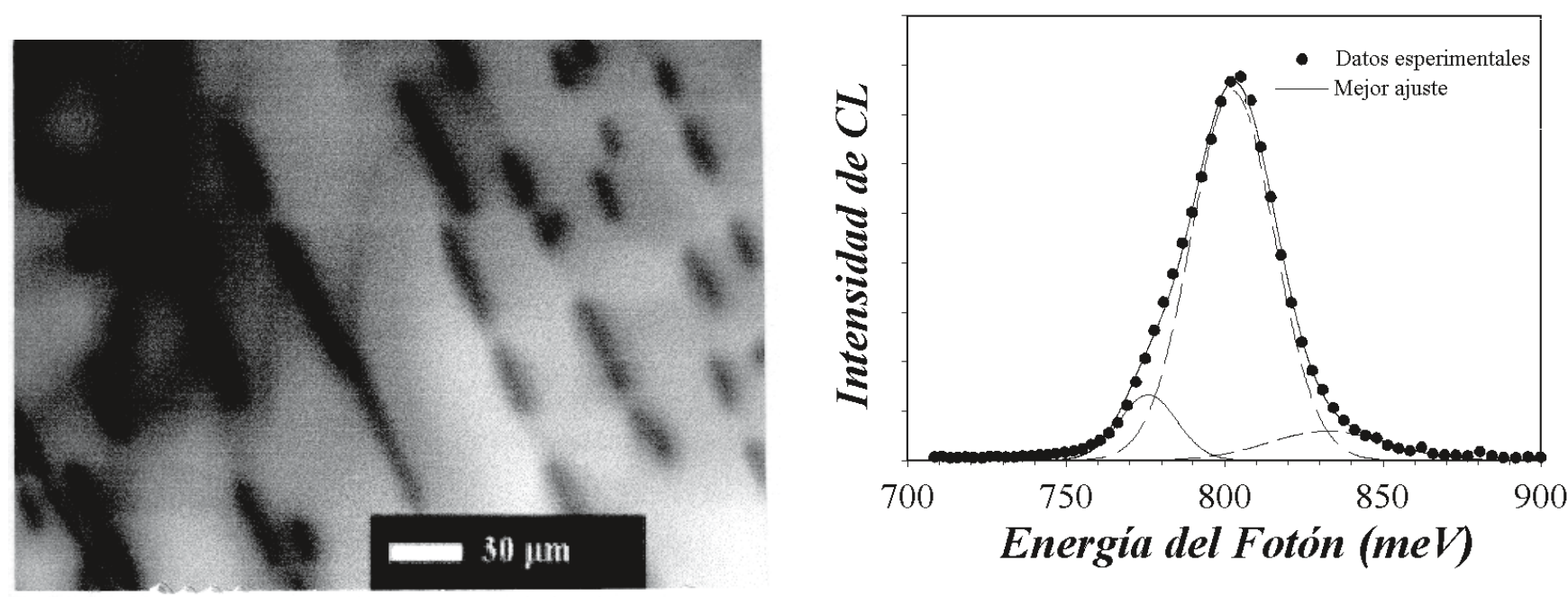

Figura 3. (a) Imagen y (b) espectro de CL de los precipitados observados en el lingote 30 dopado con la concentración mas alta de Nd. 
análogas a las del lingote 26. Por otra parte, cuando la concentración de Er aumenta por encima de dicho valor, la banda A de defectos reaparece. El espectro de CL para la muestra 28 presenta la emisión cercana al borde de banda así como la banda de defectos nativos localizada a $777 \mathrm{meV}$ que generalmente se observa en este material.

Por otra parte en la muestra 29 con una concentración de $\mathrm{Nd}$ intermedia, aparece también la banda de defectos y su intensidad es comparable a la emisión del gap de banda. En la figura 3 se presentan tanto la imagen como el espectro de CL para la muestra 30 cuya concentración inicial de $\mathrm{Nd}$ es la mas alta. En la figura $3 a$ pueden observarse los precipitados que se han presentado ya anteriormente. Por otra parte, el espectro mostrado en la figura $3 \mathrm{~b}$ muestra una pronunciada disminución de la banda de $777 \mathrm{meV}$. No obstante este efecto de disminución de la banda de defectos nativos y del aumento en la intensidad de emisión proveniente del gap se ve limitada en gran medida por los procesos de precipitación mencionados.

\section{CONCLUSIONES}

En este trabajo se ha demostrado la formación de precipitados en lingotes de GaSb dopado con Er y Nd para concentraciones iniciales de dopante iguales o superiores a 9x10 $19 \mathrm{~cm}^{-3}$. Estos precipitados presentan una alta concentración de elemento de TR respecto al material circundante. El carácter tipo p de las muestras se ve acentuado a medida que aumenta la concentración de dopante según revelan las medidas de resistividad, movilidad y densidad de portadores realizadas mediante la técnica de Van der Paw. Se ha demostrado también mediante medidas de CL la reducción de la banda de defectos debido a la presencia de los iones de Er.

\section{AGRADECIMIENTOS}

Este trabajo ha sido subvencionado por CICYT a través del proyecto ESP-98 1340.

\section{BIBLIOGRAFÍA}

1. S. Coffa, A. Polman and R. N. Schwartz, "Rare Earth Doped Semiconductors II", MRS Symp. Proc. 422 (Materials Research Society, Pittsburg, 1996).

2. "Infrared spectroscopy of Er-Containing amorphous silicon thin films", A. R. Zanatta, L. A. O. Nunes, Appl. Phys. Lett. 71 (25) 3679-3681. (1997).

3. "Photoluminescence of epitaxial InP:Yb films", L. F. Zakharenkov, V. A. Kasatkin, F. P. Kesamanly, B. E. Samorukov, M. A. Sokolova, Sov. Phys. Semicond. 15. 946. (1981)

4. "Intracenter transitions in $\mathrm{Yb}^{3+}$ impurities in Gallium Phosphide", V. A. Kasatkin, F. P. Kesamanly, V. G. Makarenkov, V. F. Masterov, B. E. Samorukov, Sov. Phys. Semicond. 14. 1092. (1980)

5. "Photoluminescence of heat-treated Gallium Phosphide doped with Prasseodimium and Ytterbium", V. A. Kasatkin, F. P. Kesamanly, B. E. Samorukov, Sov. Phys. Semicond. 15. 352-353. (1981).

6. "Trap level characteristics of rare-earth luminescence centers in III-V semiconductors", A. Taguchi, K. Takahei, J. Appl. Phys. 79 (8). 4330-4334. (1996).

7. "Effect of Erbium doping on the defect structure of GaSb crystals", P. Hidalgo, B. Méndez, J. Piqueras, J. Plaza, E. Diéguez, Semic. Sci. Technol. 13. 14311433 (1998)

8. "Electrical properties at the Nd-doped $\mathrm{Si}_{-} \mathrm{SiO}_{2}$ interface", T. J. Zhang, S. Y. Li, Sol. State Electron. 30. 775-776. (1986).

9. "Bridgman growth of GaSb with planar interface", P. S. Dutta, K. S. Sangunni, H. L. Bhat, V. Kumar, J. Cryst. Growth 144. 44-48. (1995).

10. D.T. Hurle, "Handbook of crystal growth" , vol. 2b. Cap. 12 661-705, North Holland, Amsterdam, 1994.

11. "Thermal convection and longitudinal macrosegregation in horizontal Bridgman crystal growth. (I) Order of Magnitude", D. camel. J. J. Favier, J. crystal growth 67. 42-56. (1984).

12. "Thermal convection and longitudinal macrosegregation in horizontal Bridgman crystal growth. (II) Practical laws", D. camel. J. J. Favier, J. Crystal Growth 67. 57-67. (1984)

13. "Magnetic-ion-lattice interaction: Rare earth antimonides", M. E. Mullen, B. Lüthi, P. S. Wang, E. Bucher, L. D. Longinotti and J. P. Maita, Phys. Rev. B, 10. 186-199. (1974).

14. "Native defects in the $\mathrm{Al}_{\mathrm{x}} \mathrm{Ga}_{1-\mathrm{x}} \mathrm{Sb}$ alloy semiconductor", M. Ichimura, K. Higuchi, Y. Hattori, T. Wada, N. Kitamura, J. Appl. Phys. 68. 6153-6158. (1990) 